\title{
WEIGHT REDUCTION OF A DRONE USING GENERATIVE DESIGN
}

\author{
BÁLINT LEON SEREGI*1 AND PÉter FICZERE ${ }^{1}$ \\ ${ }^{1}$ Department of Vehicle Elements and Vehicle-Structure Analysis, Budapest University of Technology and \\ Economics, Múegyetem rkp. 3, Budapest, 1111, HUNGARY
}

\begin{abstract}
Generative design has the potential to be optimized with different parameters using a design method based on artificial intelligence and by defining the design problem. The use of this method on a drone frame is presented with explanations of the various design phases. The goal of the optimisation was to be able to fit a battery with a larger capacity onto the Unmanned Aerial Vehicle and compensate for its increased weight by reducing the weight of the drone frame using a generative algorithm. As the production possibilities were limited, adaptation to the selected manufacturing technology was also taken into account during the optimization.
\end{abstract}

Keywords: generative design, additive manufacturing, weight reduction

\section{Introduction}

Mechanical design is a complex process. Engineers not only design a construction to meet functional and safety requirements, but also have to take into consideration the costs, functional expectations, safety, usage needs, lifespan and manufacturability. That is why the most optimal design, which appropriately fulfils every need, must be selected from several versions when designing a product. The spread of rapid prototyping facilitates the rapid creation and testing of these versions. In connection with this, the phenomenon of the design paradox can be observed, which concerns the relationship between product knowledge and design decisions over time as a function of the product from its design to production (Fig. 1). [1] This engineering influence has an outstanding effect on costs, mainly during the design phase and the production preparation process. If an error in the product concept is revealed during the testing phase immediately before production, its reworking increases costs significantly.

Rapid prototyping is suitable for eliminating this paradox. The main reasons for this are that it

- makes communication during the design phase with faster iterations more effective;

- reduces the development time;

- reduces the likelihood of costly errors (hidden design errors) and those following the release of the product from occurring;

- extends the product life cycle by adding required and eliminating unnecessary features early on in the design phase;

\footnotetext{
*Correspondence: seregibalint@edu.bme.hu
}

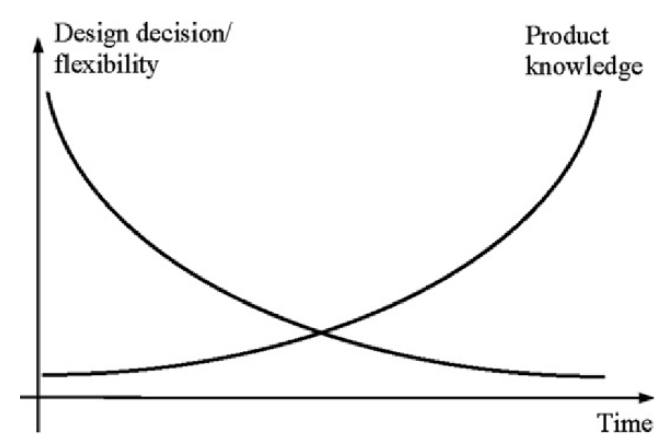

Figure 1: The design paradox[1]

- can avoid design flaws from occurring early on, even in the concept phase.

The generative design is a great solution to lower this influence by making many versions. These outcomes all meet the given requirements (if any one of them cannot be achieved, the algorithm stops as a result of a failure). Usually the generative design concerns automotive applications where one or more components are merged into one solid component, thereby also reducing the weight. This is obvious from a transportation point of view since it can reduce the environmental impact by reducing fuel consumption and using less raw materials. For instance, General Motors (GM) optimized their seat bracket, which is a standard component that fixes the seat and seat belt lock to the car's floor. While previously this bracket consisted of 8 components, generative software could come up with more than 150 organic-looking outcomes (Fig. 2 ). The chosen outcome by GM was $40 \%$ lighter and $20 \%$ stiffer than the original design. [2] 

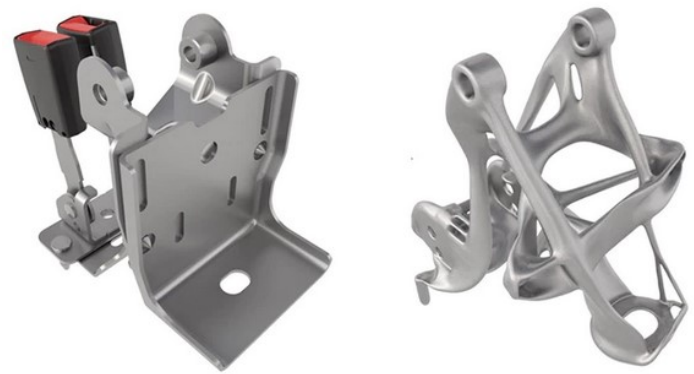

Figure 2: The original and the generative concept [2]

Additive manufacturing is very different from its predecessor, that is, subtractive manufacturing. Over the history of machining, as a result of Computer Numerical Control, technology has made great strides in terms of efficiency, productivity and accuracy. 5-axis machines as well as 6-axis robotic arms have also appeared, which in the case of the tool and material to be machined are the limiting factors. There are geometries which cannot be produced even by 6 -axis robotic arms as the tool simply does not fit in some concave areas. For harder materials that are difficult to machine, a tool made of a stronger material is required, which must be specially designed with customised machining parameters. Furthermore, depending on the amount of material deposited, the chips as loss are displayed. [3]

To demonstrate the reduction in weight as a result of generative design, a small-scale drone frame was optimized. The goal was to create a small flying Unmanned Aerial Vehicle (UAV) with a generative designed frame that reduces the overall weight of the vehicle as the maximum speed and range of UAVs strongly depend on the performance of their motors and their overall weight. The weight of the battery cannot be reduced because decreasing its capacity would also shorten its available range. Therefore, the weight of the frame had to be reduced just as a larger battery with a greater capacity would have had to have been, thereby increasing its range. Concerning the size of the motors, the acquirement of high-performing and small ones on the commercial market is preferred. Its electronics and control consist of the Electronic Speed Controller, PXFmini autopilot card and a Raspberry Pi Zero. The PXFmini autopilot card contains the basic sensors, e.g., a compass, GPS and barometer, which are connected to the onboard computer, that is, the Raspberry Pi. This electronics stack can facilitate basic functions like stabilization and RC communication.

\section{Methodology}

As the combined performance of its four motors can lift 2800 grams in total, this equates to the critical weight of the UAV during the design phase. Its weight without the frame (the electronics, motors and battery) is 404 grams. This is $1 / 7$ of the critical weight which means that the vehicle can achieve higher speeds and accelerations, moreover, the energy consumption can also be
Table 1: Mechanical parameters of ABS [4]

\begin{tabular}{lc}
\hline Young's modulus [GPa] & 2.2 \\
Tensile strength [MPa] & 30 \\
Density $\left[\mathrm{g} / \mathrm{cm}^{3}\right.$ ] & 1.1 \\
Poisson's ratio & 0.4 \\
\hline
\end{tabular}

more favourable especially with the reduced weight of the frame. The frame is composed of the plastic Acrylonitrile Butadiene Styrene (ABS), the parameters of which can be seen in Table 1. These mechanical parameters were used during its generation.

First, the basic concept must be created. The electronics can be placed in stacks as a block thanks to their dimensions (Fig. 3).

The battery was placed under the electronics stack 38 millimetres from the plane of the motors to somewhat increase its in-flight stability. During the design phase, a basic model is required to determine the preserve and obstacle geometries, loads and constraints. The preserve geometry means the volume that cannot be modified by the algorithm (Fig. 4). The loads and constraints can be determined on the following areas; in this case the mounting discs of the motors and mounting rails of the electronics. The obstacle geometry is the volume within which the algorithm is not allowed to place material (Fig. 5), since it is a placeholder for other components like bolts, batteries and motors. The constraints and loads were defined in different load cases which simulate the lift, weight force of the battery and three crash-landings from different angles. Specifying design criteria is also mandatory.

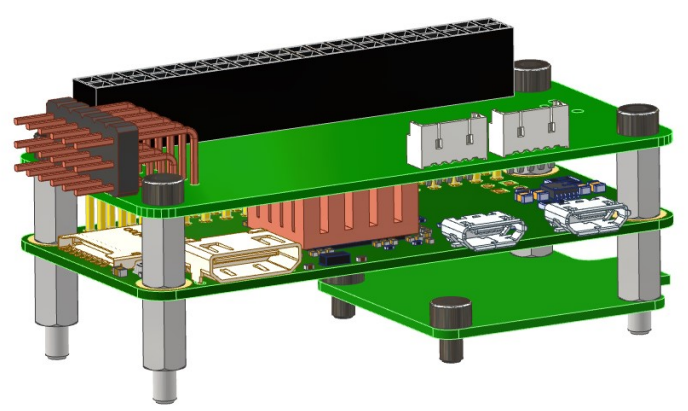

Figure 3: The electronics stack

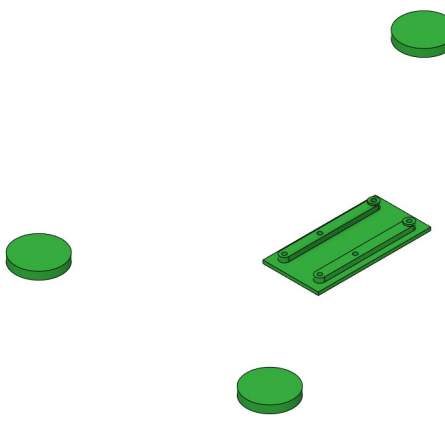

Figure 4: Preserve geometry 


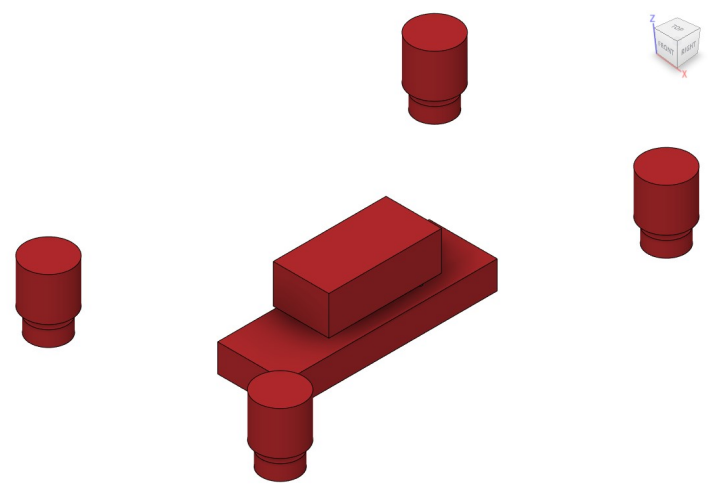

Figure 5: Obstacle geometry

Although by default the program is set to minimize the mass, it is possible to specify a specific target mass with a safety factor. In addition, the manufacturing technology can be specified to optimize the result and meet needs. The available options are $2.5-5$ axis cutting, additive fabrication, casting and indefinite. The indefinite option differs in that it does not take into account the limiting factors of the technologies such as the smallest tool diameter or tool overhang for cutting, alternatively, in the case of additive manufacturing, the manufacturing direction, orientation and maximum overhang angle of the workpiece. During one run, several technologies can be selected according to needs. The weight reduction was set with a target mass of 84 grams and safety factor of 1.2.

\section{Investigation}

Using Fusion 360's generative design algorithm, 6 different results were generated, from which the most optimal frame was chosen (Table 2). The reason for the 6 results is the possibility of different build directions (a key parameter in additive manufacturing), which the program also takes into account and the given model is once more iterated based on this. The generative model generated by the preserve geometry can be seen where the algorithm did not remove any volume and the robotic arms were reduced to smaller robotic arm braces. The amount of weight reduction made it possible to use a battery with a larger capacity that lengthens the flight time. The basic concept was fitted with a 2200 mAh battery. From this product family, the next largest capacity of the battery is $2650 \mathrm{mAh}$. The smaller and larger batteries weigh 168 grams and 232 grams, respectively. Compared to the orig-

Table 2: Parameters of the outcomes

\begin{tabular}{ccc}
\hline Mass [kg] & Max. diplacement $[\mathrm{mm}]$ & Volume $\left[\mathrm{mm}^{3}\right]$ \\
\hline 0.079 & 3.15 & 74962.69 \\
0.073 & 5.11 & 68565.46 \\
0.136 & 2.98 & 128678.19 \\
0.135 & 2.42 & 127491.83 \\
0.23 & 1.76 & 217371.08 \\
0.135 & 2.43 & 127823.45 \\
\hline
\end{tabular}
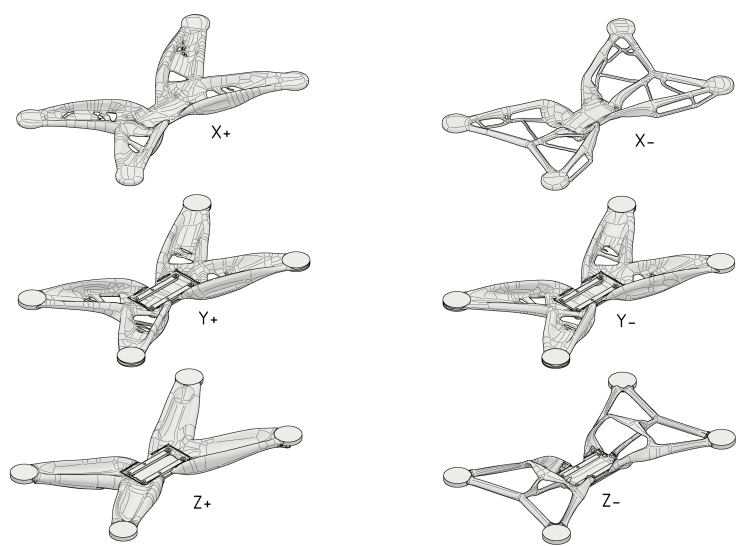

Figure 6: Outcomes of the different orientations

inal design, its overall weight was reduced by 25 grams, as the difference in weight between the two batteries is smaller than the weight reduction as a result of changing the frame, resulting in the flight time increasing by about 65 seconds (Fig. 7).

\section{Summary}

The reduction in the weight of a drone as a result of generative design was presented (the full assembly can be seen in Fig. 8). By following this method, a more efficient concept could be created. Proper use of generative design helps to develop a new or existing concept in many areas. Reducing the weight of vehicles has always been an important aspect of the industry, so further reductions in this field are expected. [5] E-mobility can also be greatly beneficial as the biggest problem is always the size and weight of batteries, which has a big impact on fuel consumption and range. Although the weight reduction of passenger cars is more complex, the example presented illustrates that this could be a revolutionary solution for the automotive industry in the future. From another point of view, it is also important to recognise that increased computational capacities make it possible to compare many more types of concepts, thereby providing the opportunity to choose the most optimal solution when designing.

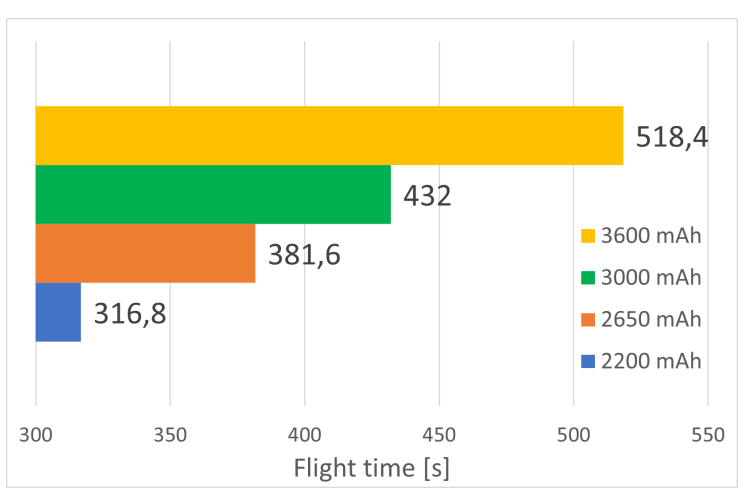

Figure 7: Flight times with different battery capacities 


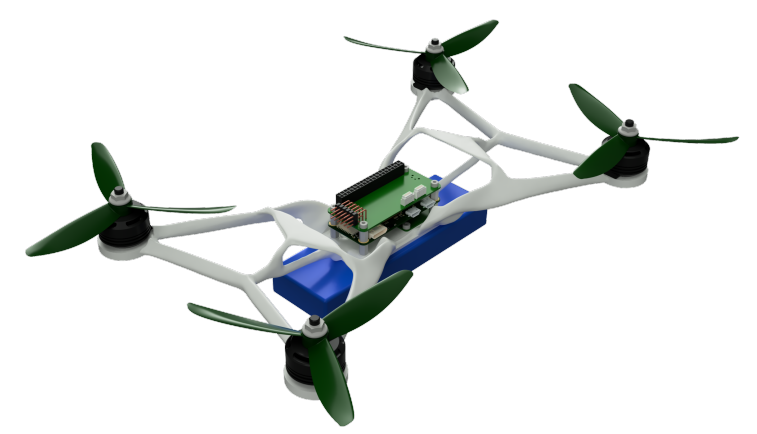

Figure 8: Assembly of the drone

\section{Additional questions that arise}

It can be seen that the results are free form surfaces of organic forms that are aesthetically pleasing which also make a positive contribution to the concept. Nevertheless, it is noticeable that although the given loads were symmetrical in all directions, the frame did not become completely symmetrical. This can be eliminated by postmirroring the model, however, the question is will the mirrored construct be equally effective?

\section{Acknowledgements}

The authors would like to express their gratitude to Arkance Systems HU Kft. for supporting this work by providing pieces of software.

\section{REFERENCES}

[1] Chang, K.H.: Product Performance Evaluation using CAD/CAE (Academic Press), 2013. ISBN: 0123984696

[2] Alderton, M.: Driving a lighter, more efficient future of automotive part design, 2021. https://www.autodesk.com/customer-stories/general-motorsgenerative-design

[3] Siva Rama Krishna, L.; Srikanth, P.: Evaluation of environmental impact of additive and subtractive manufacturing processes for sustainable manufacturing. Mater. Today: Proc., 2021, 45(2), 3054-3060, international Conference on Advances in Materials Research - 2019. DOI: 10.1016/j.matpr.2020.12.060

[4] Martinetti, A.; Margaryan, M.; van Dongen, L.: Simulating mechanical stress on a micro Unmanned Aerial Vehicle (UAV) body frame for selecting maintenance actions. Procedia Manuf., 2018, 16, 6166, Proceedings of the 7th International Conference on Through-life Engineering Services. DOI: 10.1016/j.promfg.2018.10.160

[5] Shahrubudin, N.; Lee, T.; Ramlan, R.: An Overview on 3D Printing Technology: Technological, Materials, and Applications. Procedia Manuf., 2019, 35, 1286-1296, The 2nd International Conference on Sustainable Materials Processing and Manufacturing, SMPM 2019, 8-10 March 2019, Sun City, South Africa. DOI: 10.1016/j.promfg.2019.06.089 